\title{
Hypothesis \\ Tunable and Sensitive Detection of Cortisol Using Anisotropic Phosphorene with a Surface Plasmon Resonance Technique: Numerical Investigation
}

\author{
Vipin Kumar Verma ${ }^{1,2}$, Sarika Pal ${ }^{1}$, Conrad Rizal ${ }^{3}$ iD and Yogendra Kumar Prajapati ${ }^{4, *}$ (D) \\ 1 ECE Department, National Institute of Technology Uttarakhand, Srinagar 246174, India; \\ vipin.phd20@nituk.ac.in (V.K.V.); sarikapal@nituk.ac.in (S.P.) \\ 2 KIET Group of Institutions, Delhi-NCR, Ghaziabad 201206, India \\ 3 Seed NanoTech International Inc., Brampton, ON L6Y 3J6, Canada; conrad.rizal@seed-nanotech.com \\ 4 ECE Department, Motilal Nehru National Institute of Technology Allahabad, Prayagraj 211004, India \\ * Correspondence: yogendrapra@mnnit.ac.in
}

check for

updates

Citation: Verma, V.K.; Pal, S.; Rizal

C.; Prajapati, Y.K. Tunable and

Sensitive Detection of Cortisol Using Anisotropic Phosphorene with a

Surface Plasmon Resonance

Technique: Numerical Investigation.

Magnetochemistry 2022, 8, 31.

https://doi.org/10.3390/

magnetochemistry 8030031

Academic Editors: Roberto Zivieri and Evgeny Katz

Received: 28 November 2021

Accepted: 2 March 2022

Published: 7 March 2022

Publisher's Note: MDPI stays neutral with regard to jurisdictional claims in published maps and institutional affiliations.

Copyright: (C) 2022 by the authors. Licensee MDPI, Basel, Switzerland. This article is an open access article distributed under the terms and conditions of the Creative Commons Attribution (CC BY) license (https:// creativecommons.org/licenses/by/ $4.0 /)$.

\begin{abstract}
Tunable and ultrasensitive surface plasmon resonance (SPR) sensors are highly desirable for monitoring stress hormones such as cortisol, a steroid hormone formed in the human body's adrenal glands. This paper describes the detection of cortisol using a bimetallic SPR sensor based on a highly anisotropic two-dimensional material, i.e., phosphorene. Thicknesses of bi-metal layers, such as copper $(\mathrm{Cu})$ and nickel $(\mathrm{Ni})$, are optimized to achieve strong SPR excitation. The proposed sensor is rotated in-plane with a rotation angle $(\varphi)$ around the $z$-axis to obtain the phosphorene anisotropic behavior. The performance parameters of the sensor are demonstrated in terms of higher sensitivity $\left(347.78^{\circ} / \mathrm{RIU}\right)$, maximum angular figure of merit $\left(\mathrm{FOM}^{*}=1780.3\right)$, and finer limit of detection $(0.026 \mathrm{ng} / \mathrm{mL})$. Furthermore, a significant penetration depth $(203 \mathrm{~nm})$ is achieved for the proposed sensor. The obtained results of the above parameters indicate that the proposed sensor outperforms the previously reported papers in the literature on cortisol detection using the SPR technique.
\end{abstract}

Keywords: anisotropy; angular FOM*; the limit of detection; phosphorene; sensitivity; surface plasmon resonance

\section{Introduction}

Cortisol is a steroid hormone and is commonly referred to as the stress hormone because of its connection to the stress response of human beings. The cortisol level in blood and saliva impacts the cardiovascular processes, blood pressure, and many other metabolic activities [1]. For example, a rise in cortisol level may cause Cushing syndrome-a fatty hump between shoulders, a rounded face, and various stretch marks on the skin. In contrast, cortisol insufficiency can cause Addison disease-an uncommon disorder that occurs when the body cannot produce enough of certain hormones [1]. Measuring cortisol levels is necessary for determining its deficiency, saturation levels and identifying various disorders linked to it. Cortisol levels can be determined using a variety of body fluids, including blood (invasive) and saliva (non-invasive) [1,2]. Its status in the saliva is straightforward to measure because these samples keep their original properties for at least a week or more [1]. SPR sensors are more suited than other mentioned sensors due to their quick, real-time, and label-free sensing capabilities [3]. The SPR sensor is a powerful tool that detects molecular interactions by changing the probing medium's refractive index (RI).

To study the level of cortisol in saliva, Steven et al. reported a six-channel portable SPR biosensor based on competition assays [1]. The cortisol detection limit $(0.36 \mathrm{ng} / \mathrm{mL})$ in laboratory buffers was determined. Furthermore, to detect cortisol levels, researchers proposed and used gold $(\mathrm{Au})$ nanoparticle-based long-range surface plasmon resonance 
(LRSPR) sensors, grating-based fiber optic sensors, and lossy mode resonance-based SPR sensors [3-7]. Some of the critical experimental works for cortisol detection reported in the literature are based on techniques such as electrochemical immuno-sensor, aptamer-based Au nanoparticles, and SPR immunoassay. However, the limit of detection (LOD) values using these techniques are still lower $(\leq 1 \mathrm{ng} / \mathrm{mL})$, respectively [6-8]. M. Frasconi et al. examined an SPR-based sensor for real-time cortisol and cortisone detection in saliva and urine samples with a detection limit of $10 \mu \mathrm{g} / \mathrm{L}$ [9]. S. Jo et al., demonstrated the use of an Au nanoparticle-based localized surface plasmon resonance (LSPR) sensor to create a highly sensitive and selective cortisol sensor experimentally [10]. The suggested LSPR biosensor with an immobilized aptamer demonstrated a detection limit of $0.1 \mathrm{~nm}$ for cortisol concentrations ranging from 0.1 to $1000 \mathrm{~nm}$. C. Leitao et al., recently suggested a cortisol immunosensor based on SPR and reached a detection limit of $1 \mathrm{pg} / \mathrm{mL}$ using AuPd plasmonic unclad plastic optical fiber (POF) [11].

Recently researchers demonstrated the improved performance of the SPR sensor by utilizing the two-dimensional (2-D) materials such as graphene, transition metal dichalcogenides (TMDs), few layers of phosphorene-a monolayer of black phosphorus (BP), antimonene, and $\mathrm{Ti}_{3} \mathrm{C}_{2} \mathrm{~T}_{\mathrm{x}}$-Mxene over a metal layer due to their high charge carrier mobility, large work function, high adsorption energy, large surface area, stronger interaction with biomolecules, and high chemical stability [12-16]. Therefore, 2-D materials have a high potential for sensing, photonic, and optoelectronic applications; however, use in SPR sensors for cortisol detection remains elusive. However, the zero bandgaps of graphene, low carrier mobility, and hydrophobicity of TMDs, as well as the narrow energy bandgap of MXene became their fatal disadvantages [17]. Alternatively, phosphorene is a stable 2-D layered material possessing excellent hole mobility $\left(10,000 \mathrm{~cm}^{2} \cdot \mathrm{V}^{-1} \cdot \mathrm{s}^{-1}\right)$, tunable bandgap $(0.3-2 \mathrm{eV})$, attention-grabbing puckered surface morphology, strong binding energy, hydrophilic nature, 40 times higher molar response factor (even more significant than graphene and TMDs), and parts per billion (ppb) sensing ability and have shown great potential for gas, humidity, and biosensing [18-22]. Thus, phosphorene's sensitivity, $\mathrm{S}$, and selectivity for water vapors and gas can be tuned to advantage when used for sensing cortisol concentration in saliva. However, long-time direct exposure of phosphorene to the ambient environment may lead to its oxidation [16]. So, care must be taken during the experimental process using a high-quality and stable phosphorene sheet to obtain accurate results.

The most exceptional property of phosphorene is its in-plane anisotropy arising from its $\mathrm{sp}^{3}$ hybridized puckered lattice structure [23]. Utilizing the in-plane anisotropy feature of phosphorene to produce a tunable sensor device, this work aims to enhance the sensor performance in terms of sensitivity and LOD for detecting cortisol concentration at an operating wavelength, $\lambda=830 \mathrm{~nm}$ [3]. However, for the tunability aspect, the strongest excitation of plasmons on the metal-phosphorene interface is considered through the rotation angle $(\varphi)$ of the integrated device around the $z$-axis in-plane, resulting in variable charge transfer between phosphorene and the metal, leading to change in minimum reflectivity. In a broader sense, the objective of this paper is to demonstrate tunable sensitivity by simply rotating the integrated device around the $z$-axis in-plane.

More importantly, the uniqueness of this work is twofold. First, we have proposed a sensor for cortisol concentration detection with enhanced performance parameters (sensitivity, LOD, etc.) over the existing state-of-the-art methods. Second, we have introduced and analyzed the influence of the anisotropic behavior of phosphorene to enhance the charge transfer between phosphorene and the metal layer, which results in the finest angular figure of merit $\left(\mathrm{FOM}^{*}\right)$ over the other existing cortisol concentration sensors.

\section{Theoretical Modeling and Performance Parameters}

Figure 1 shows the proposed Kretschmann configured SPR sensor for cortisol concentration sensing. It consists of BK-7 prism, bi-metal layers $(\mathrm{Cu} / \mathrm{Ni})$, phosphorene, and cortisol saliva solutions. The working principle of the proposed sensor is based on sur- 
face plasmon resonance conditions. Surface plasmons (SPs) are quanta of charge density oscillations at the metal/dielectric interface that are excited by incident p-polarized light with coupling through a prism, grating, or waveguide $[3,13-15,24]$. Prism coupling is a preferred technique for its realizable and straightforward geometry [3,14], where the resonance condition can be achieved after matching of wave vector of incident light with the wave vector of surface plasmon wave (SPW) $\left(\mathrm{k}_{\mathrm{inc}}=\mathrm{k}_{\mathrm{spw}}\right)$. This matching condition is susceptible to changes in the refractive index (RI) of the probing media due to adsorption of analyte on the sensor surface, which may be exploited for imaging and sensing applications [14]. Here, a low RI prism is chosen for enhanced light coupling [25]. The two combinations of bi-metal layers are used to identify the best metal layers for enhanced sensitivity. Salivary solutions containing different cortisol concentrations are considered an analyte medium where the RI values at different cortisol concentrations are taken from R.C. Stevens et al. [1] The RIs and optimized thicknesses of all constituent layers are presented in Table 1.

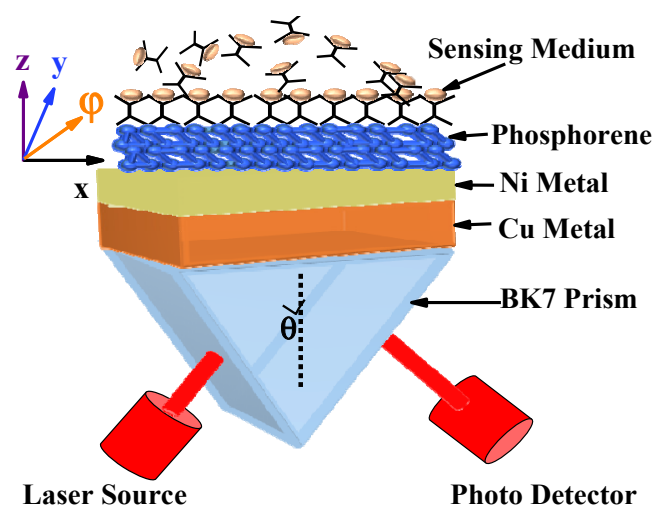

Figure 1. Proposed SPR sensor configuration in attenuated total internal reflection mode.

Table 1. Proposed sensor's thickness and RIs of constituent layers at the characteristic wavelength at $\lambda=830 \mathrm{~nm}$.

\begin{tabular}{ccc}
\hline Constituent Layers & Thickness $\mathbf{( n m )}$ & Refractive Index (RI) \\
\hline BK-7 Prism & - & $1.5102[15]$ \\
Copper layer & 15 & $0.10807+i \times 5.3990[25]$ \\
Nickel layer & 80 & $2.2777+i \times 5.0030[25]$ \\
& $6 \times 0.53$ & Optimized by tuning with the rotation \\
Phosphorene (BP) & angle $(\varphi)$ of the sensor around the \\
& & $z$-axis [23] \\
\hline
\end{tabular}

The RIs of all isotropic layers are fixed except for phosphorene, whose RI is directionaldependent due to its anisotropic nature. Phosphorene-a monolayer of black phosphorus (BP) - is an orthorhombic layered crystal with two optical axes: $C_{1}$ and $C_{2}$, whose biaxial dielectric coordinate system does not lie parallel to the crystal coordinate subsystem [23]. In order to obtain the strongest SPR excitation, a well-matched condition between two optical axes of phosphorene and SPR wave is needed. One way to achieve this is by tuning the rotation angle, $\varphi$, of the SPR device around the $z$-axis in the plane. Once the angle $\varphi$ is fixed, incident angle $\theta$ is varied from 1 to $90^{\circ}$, and RI of phosphorene is calculated using transfer matrix method (TMM) modeling proposed in Ref. [23]. The TMM is an accurate and popular method used to calculate the reflected intensity of p-polarized incident light $[12,13]$. The TMM for the reflectance calculation of the proposed sensor has been discussed in the supplementary material (SM1). In the present case, we have used MATLAB software to simulate the results (i.e., to obtain reflectance curves) and analyze the sensor performance analytically in terms of sensitivity $\left(S=\frac{\Delta \theta_{S P R}}{\Delta n_{a}}\left[{ }^{\circ} / R I U\right]\right)$, minimum reflectance $\left(\mathrm{R}_{\mathrm{min}}\right)$, full width at half maximum (FWHM), detection accuracy $(\mathrm{DA}=1 / \mathrm{FWHM})$, the limit of detection $\left(\mathrm{LOD}=\frac{\Delta \mathrm{C}_{\mathrm{Conc}}}{\Delta \theta_{\mathrm{SPR}}} \times 0.001^{\circ}[\mathrm{ng} / \mathrm{mL}]\right)$, figure of merit 
$\left(\mathrm{FOM}=\mathrm{S} \times \mathrm{DA}\left[\mathrm{RIU}^{-1}\right]\right)$, and angular figure of merit $\left(\mathrm{FOM}^{*}=\left|\frac{\mathrm{dR}(\theta))\left(\mathrm{dn}_{\mathrm{a}}\right)}{\mathrm{R}(\theta)}\right|[23,24]\right.$, here, * indicates the angular word, and the angular detection limit of $0.001^{\circ}$ is taken for the angular interrogated SPR sensor [24]. Note that FWHM measures the angular width of the SPR curve at $50 \%$ reflection intensity, i.e., $R_{\min }=0.5$ a.u.

\section{Results and Discussion}

\subsection{Metal Thickness Optimization and Reflectance Curves}

Several previous studies reveal that the metal layer plays a significant role in the generation of surface plasmons (SPs) and the attachment of analytes during SPR sensing applications $[23,24]$. However, the use of single metal shows less sensitivity due to poor attachment of analyte on it, which can be further enhanced by using bi-metal layers. According to the literature, using a bi-metal layer combination in the SPR sensor results in higher sensitivity and accuracy [25]. Moreover, optimizing metal layer thicknesses is essential to achieve the nearly minimum reflectance $\left(R_{\min }\right)$ value at the resonance angle for attaining high DA and angular FOM*. Therefore, we have verified the sensor performance in maximal sensitivity and $\mathrm{R}_{\min }$ for a few $\mathrm{Cu} / \mathrm{Ni}$ metal layer thickness combinations. For example, the metal thickness combination of $\mathrm{Cu} / \mathrm{Ni}$ is $40 / 35 \mathrm{~nm}$, meaning that the thickness of $\mathrm{Cu}$ and $\mathrm{Ni}$ is $40 \mathrm{~nm}$ and $35 \mathrm{~nm}$, respectively.

Figure 2 illustrates how to optimize the $\mathrm{Cu} / \mathrm{Ni}$ thickness at monolayer anisotropic phosphorene in terms of sensitivity and Rmin by balancing photon absorption energy and electron energy loss [23]. As illustrated in Figure 2, the addition of $\mathrm{Ni}$ to the $\mathrm{Cu}$ layer increases sensitivity, which is not achievable with a single metal layer. Propagation of SPR waves in phosphorene, unlike isotropic 2D nanomaterial (e.g., graphene, TMDs), is significantly different [20]. Hence, the strongest SPR excitation can be achieved only after perfect matching optical axes of phosphorene crystal with p-polarized incident light by tuning the rotation angle $(\varphi)$ [23]. In this regard, the $\varphi$ is optimized for each thickness combination of $\mathrm{Cu} / \mathrm{Ni}$ by rotating the proposed sensor around the $z$-axis. Figure 2 plotted at the optimized value of $\varphi$, i.e., $72^{\circ}$, depicts the highest sensitivity of $229.18^{\circ} / \mathrm{RIU}$ at smaller $R_{\min }=0.0612$ for the thickness of $\mathrm{Cu}(15 \mathrm{~nm})$, Ni $(80 \mathrm{~nm})$, and monolayer BP. Further, the influence of $\varphi$ on performance for the proposed sensor has been discussed in the supplementary material (SM2: Figures S1-S11).

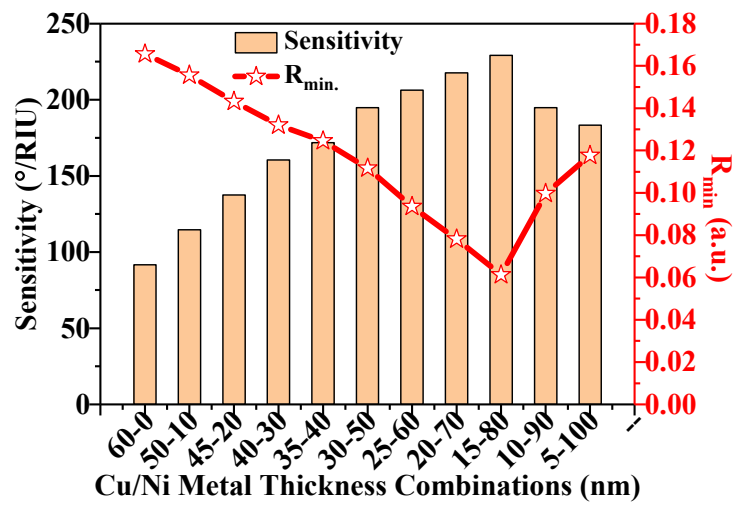

Figure 2. Bi-metal layer thickness optimization curve: sensitivity and $R_{\min }$ vs. bilayer thickness.

In contrast, sensitivity lies below this for all other thickness combinations of $\mathrm{Cu}$ and $\mathrm{Ni}$. The optical anisotropy of BP can be effectively tuned by adjusting the number of BP layers, as the optical conductivity of BP layers changes by varying its thickness [17-19]. After optimizing the thickness combination of bimetal layers of the proposed sensor, we evaluated the sensor performance parameters such as $\mathrm{S}, \mathrm{DA}$, and figure of merit (FOM) for 0 to 6 layers of BP, as shown in Table 2. It may be seen in Table 2 that all performance parameters increase with the number of BP layers. For example, the maximum sensitivity $\left(320.86^{\circ} / \mathrm{RIU}\right), \mathrm{DA}\left(0.70421 /^{\circ}\right)$, and FOM $\left(225.96 \mathrm{RIU}^{-1}\right)$ are obtained for six layers of BP. 
The improved performance is due to light-matter solid interaction observed at higher BP layers because of more significant adsorption energy and optical conductivity.

Table 2. Sensor parameters evaluated for 0-6 layers of BP.

\begin{tabular}{|c|c|c|c|c|c|c|c|}
\hline $\begin{array}{c}\text { BP } \\
\text { Layers }\end{array}$ & $\theta_{\text {res. }}$ at $n_{s}=1.33\left({ }^{\circ}\right)$ & $\theta_{\text {res. }}$ at $\mathrm{n}_{\mathrm{s}}=1.3305\left(^{\circ}\right)$ & $\mathbf{R}_{\min }$ (a.u.) & S ( ${ }^{\circ} /$ RIU) & FWHM $\left(^{\circ}\right)$ & $\operatorname{DA}\left(1 /{ }^{\circ}\right)$ & $\begin{array}{c}\text { FOM } \\
\left(\text { RIU }^{-1}\right)\end{array}$ \\
\hline 1 & 76.7935 & 76.6789 & 0.0612 & 229.18 & 1.89 & 0.5291 & 121.26 \\
\hline 2 & 77.0972 & 76.9826 & 0.0580 & 240.56 & 1.87 & 0.5348 & 128.64 \\
\hline 3 & 77.4353 & 77.3092 & 0.0506 & 252.10 & 1.80 & 0.5556 & 140.06 \\
\hline 4 & 77.8134 & 77.6816 & 0.0417 & 263.56 & 1.78 & 0.5618 & 148.07 \\
\hline 5 & 78.2374 & 78.0942 & 0.0037 & 286.48 & 1.69 & 0.5917 & 169.51 \\
\hline 6 & 78.7157 & 78.5553 & 0.0252 & 320.86 & 1.42 & 0.7042 & 225.96 \\
\hline
\end{tabular}

Further, the increment of BP layers shows higher $\mathrm{R}_{\min }$ for the proposed sensor. So, the parameters are evaluated only for 0-6 layers of BP. Therefore, all subsequent simulations for cortisol sensing are performed at optimized thicknesses of $\mathrm{Cu}(15 \mathrm{~nm}), \mathrm{Ni}(80 \mathrm{~nm})$, and six layers of BP.

The reflectance curve is simulated for different cortisol concentrations, i.e., $0.36,0.72$, $1.80,3.60$, and $4.50 \mathrm{ng} / \mathrm{mL}$ at optimized $\mathrm{Cu}(15 \mathrm{~nm})$ and $\mathrm{Ni}(80 \mathrm{~nm})$ thicknesses, and six layers of anisotropic phosphorene.

Figure 3 shows the reflectance curve at various cortisol concentrations $(0.36$ to $4.6 \mathrm{ng} / \mathrm{mL})$. As shown, the resonance angle shifts to a higher value and $R_{\min }$ also obtains more significant for higher cortisol concentrations. The improvement is due to higher binding energy and larger surface area offered due to puckered surface morphology of phosphorene for efficient adsorption of cortisol present in saliva $[19,23]$. The variation in RI of the analyte medium with adsorption of cortisol modifies the matching condition of wave vector of evanescent field and SPW at some other higher incident angle.

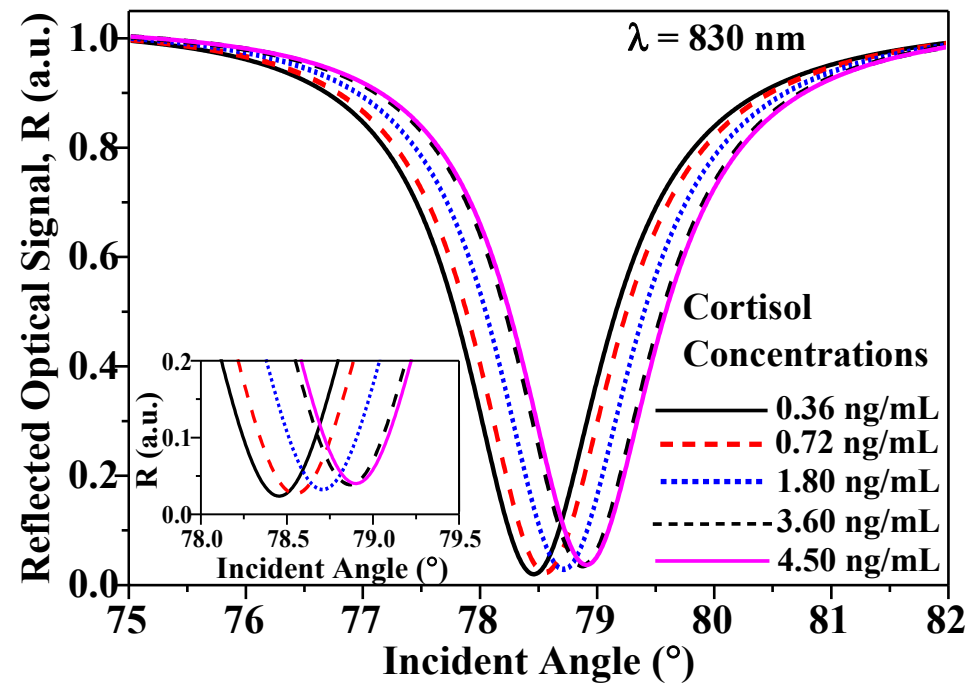

Figure 3. SPR curves for different cortisol concentrations in saliva. The inset shows the enlarged view between the incident angle 78 and $79.5^{\circ}$.

Figure 4 illustrates the variation of SPR angle, $R_{\min }$, and FWHM estimated from the reflectance curves in Figure 3. The increase in SPR angle and larger values of $R_{\min }$ for higher cortisol concentrations indicate the sensitive detection of cortisol concentration in saliva [26]. The smaller FWHM is observed at higher cortisol concentrations due to the minimum damping of SPs [23]. Likewise, as shown in Figure 5, cortisol concentrations versus sensitivity and FOM are also evaluated using reflectance curves in Figure 3 . The 
cortisol concentration of $0.36 \mathrm{ng} / \mathrm{mL}$ is used as a reference sample for sensitivity and FOM calculations. The rising trend in sensitivity and FOM is related to the sensitive and accurate detection of cortisol concentrations in saliva. A precise observation from Figure 5 indicates

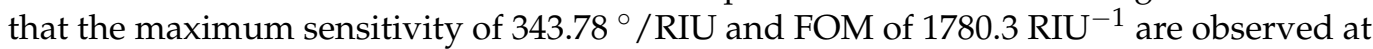
$4.5 \mathrm{ng} / \mathrm{mL}$ of cortisol concentration.

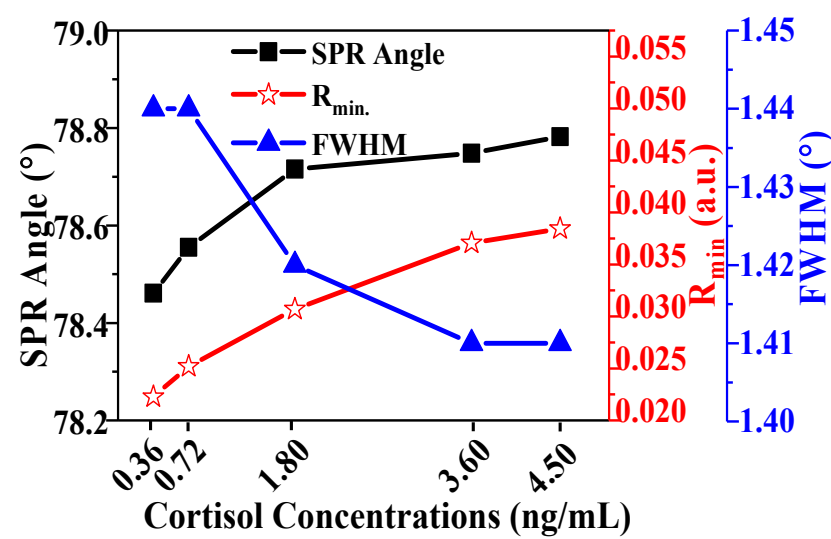

Figure 4. SPR angle, $\mathrm{R}_{\min }$, and FWHM for different cortisol concentrations ( 0.36 to $4.5 \mathrm{ng} / \mathrm{mL}$ ).

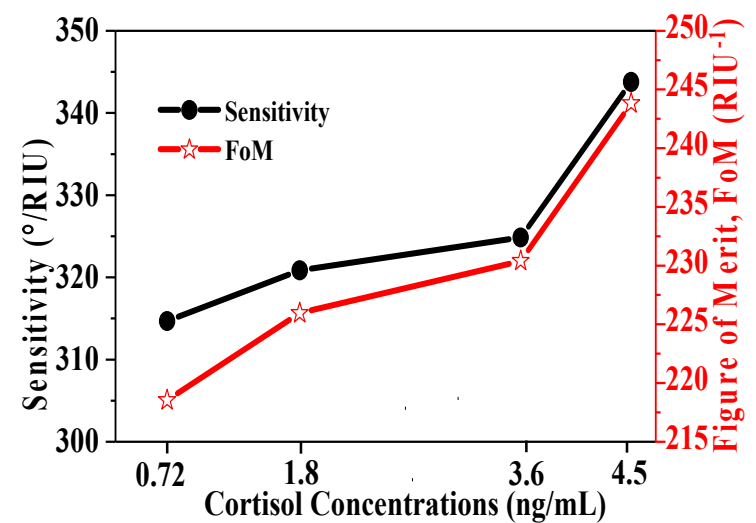

Figure 5. Sensitivity and FOM for different cortisol concentrations ( 0.72 to $4.5 \mathrm{ng} / \mathrm{mL}$ ).

\subsection{Analysis of FOM* and $L O D$}

LOD and $\mathrm{FOM}^{*}$ are two critical parameters to indicate the most exemplary performance of a proposed sensor for cortisol concentration sensing. First, the FOM ${ }^{*}$ curve slope tells the maximum value of FOM* [24]. Second, LOD signifies the lowest possible cortisol concentration that can be detected. In summary, the maximum FOM* and the smallest possible LOD value are desired to enhance cortisol concentration sensing [24].

FOM $^{*}$ curve at various cortisol concentrations is depicted in Figure 6. As shown in it, the maximum FOM* values obtained for cortisol concentrations of $0.36,0.72,1.80,3.60$, and $4.50 \mathrm{ng} / \mathrm{mL}$ are $777.8,916.9,1115.3,1313.4$, and 1780.3, respectively; highest sensitivity for a cortisol concentration of $4.50 \mathrm{ng} / \mathrm{mL}$. A much higher maximum $\mathrm{FOM}^{*}$ value indicates high accuracy ultrasensitive cortisol concentration detection. In addition to maximum FOM*, the calculated LOD for different cortisol concentrations of $0.72,1.80,3.60$, and $4.50 \mathrm{ng} / \mathrm{mL}$ are $0.0038,0.0067,0.0554$, and 0.0262, respectively, and plotted in Figure 7. 


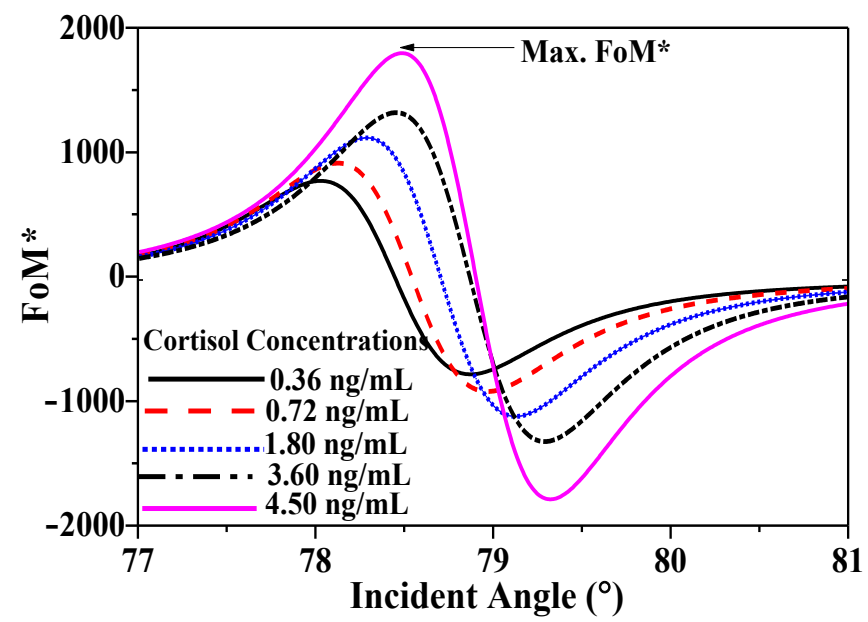

Figure 6. The angular figure of merit $\left(\mathrm{FOM}^{*}\right)$ plotted against the incident angle for different cortisol concentrations ( 0.36 to $4.5 \mathrm{ng} / \mathrm{mL}$ ).

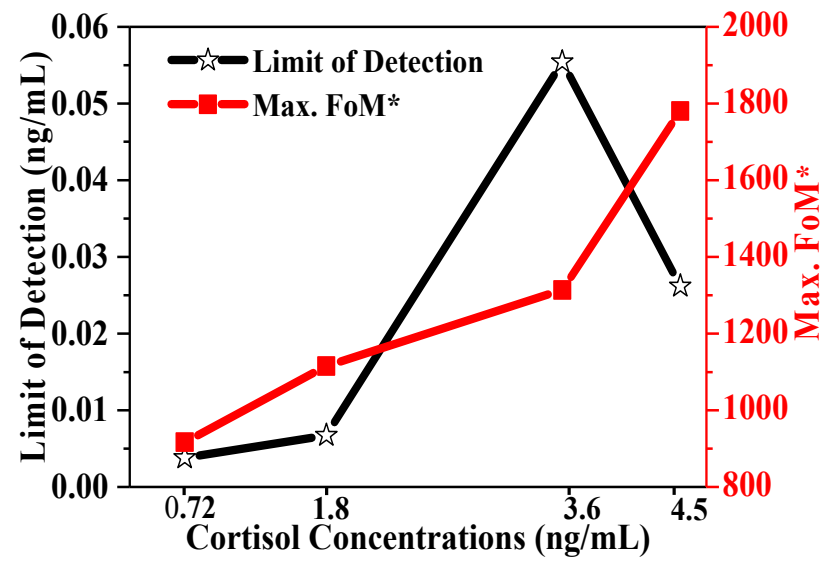

Figure 7. LOD and maximum FOM* for different cortisol concentrations $(0.72$ to $4.5 \mathrm{ng} / \mathrm{mL})$.

Table 3 depicts all the performance parameters evaluated at considered cortisol concentrations in Figures 3-7. The best performance parameters obtained are sensitivity $\left(343.78^{\circ} / \mathrm{RIU}\right)$, FOM $\left(243.82 \mathrm{RIU}^{-1}\right)$, maximum FOM ${ }^{*}(1780.30)$, and finest LOD $(0.026 \mathrm{ng} / \mathrm{mL})$ for the proposed sensor.

Table 3. Performance parameters at different cortisol concentrations.

\begin{tabular}{ccccccc}
\hline $\begin{array}{c}\text { Cortisol } \\
\text { Conc. } \\
(\mathbf{n g} / \mathbf{m L})\end{array}$ & $\boldsymbol{\theta}_{\text {SPR }}\left(^{\circ}\right)$ & FWHM $\left(^{\circ}\right)$ & S( ${ }^{\circ} /$ RIU) & $\begin{array}{c}\text { FOM } \\
(\mathbf{1} / \mathbf{R I U})\end{array}$ & $\begin{array}{c}\text { LOD } \\
(\mathbf{n g} / \mathbf{m L})\end{array}$ & $\begin{array}{c}\text { Maximum } \\
\text { FOM }^{*}\end{array}$ \\
\hline 0.36 & 78.46 & 1.44 & - & - & - & \\
0.72 & 78.56 & 1.44 & 314.67 & 218.52 & 0.0038 & 916.9 \\
1.80 & 78.72 & 1.42 & 320.86 & 225.96 & 0.0067 & 1115.3 \\
\hline 3.60 & 78.75 & 1.41 & 324.86 & 230.40 & 0.0554 & 1313.4 \\
4.50 & 78.78 & 1.41 & 343.78 & 243.82 & 0.0262 & 1780.3 \\
\hline
\end{tabular}

Furthermore, we also evaluated the normalized electric field component for the proposed SPR sensor using COMSOL simulation software. Figure 8 illustrates the distribution of the normalized electric field $\left(E_{z}\right)$ component of the evanescent field for the proposed sensor. According to Figure 8a, the maximum excitation of SPs occurs at the anisotropic $\mathrm{BP} /$ sensing layer interface and decays exponentially away from the interface [27]. Figure $8 \mathrm{~b}$ represents the zoomed-in portion of the rectangular window marked in Figure 8a to demon- 
strate the explicit representation of constituent layers of the proposed sensor. The normalized electric field component $\left(\mathrm{E}_{\mathrm{y}}\right)$ normal to the interface for the proposed SPR sensor is also plotted in Figure 9. It signifies the field distribution at each of the constituent layers' interfaces. The inset diagram within Figure 9 demonstrates the peak normalized field intensity at the anisotropic $\mathrm{BP} /$ sensing layer interface, which decays exponentially away from the sensing interface. The penetration depth (PD) measures how deeply the field penetrates the sensing region and is defined as the distance covered by the electric field from its peak value to 1 /e or $0.37 \%$ of its peaks value along normal to the interface [25]. The PD evaluated for the proposed sensor is $203 \mathrm{~nm}$ and is sufficient to detect the small size cortisol molecule [1].

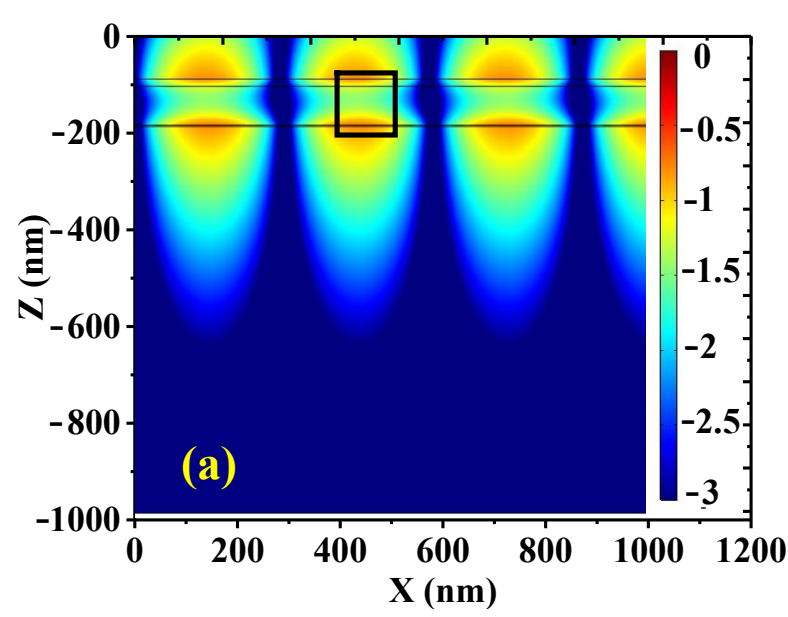

(a)

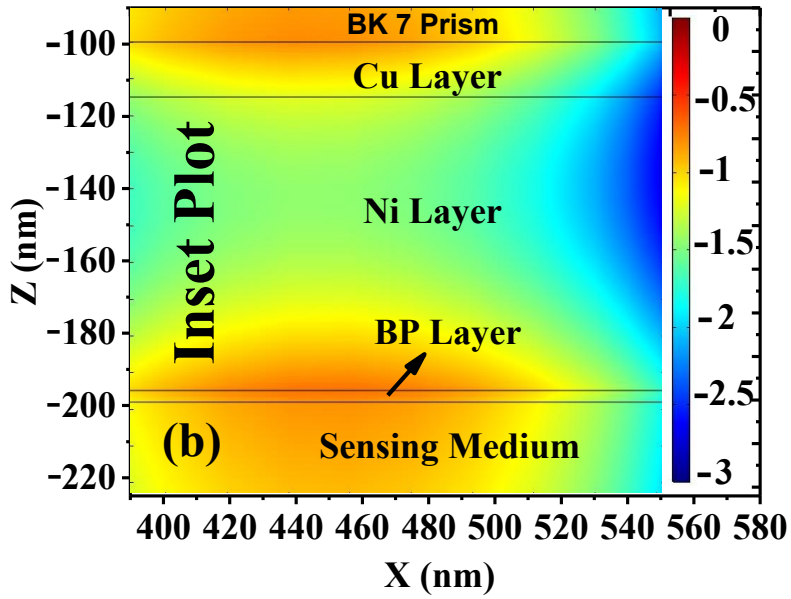

(b)

Figure 8. Proposed sensor: (a) SPs field distribution; (b) inset plot (an enlarged view).

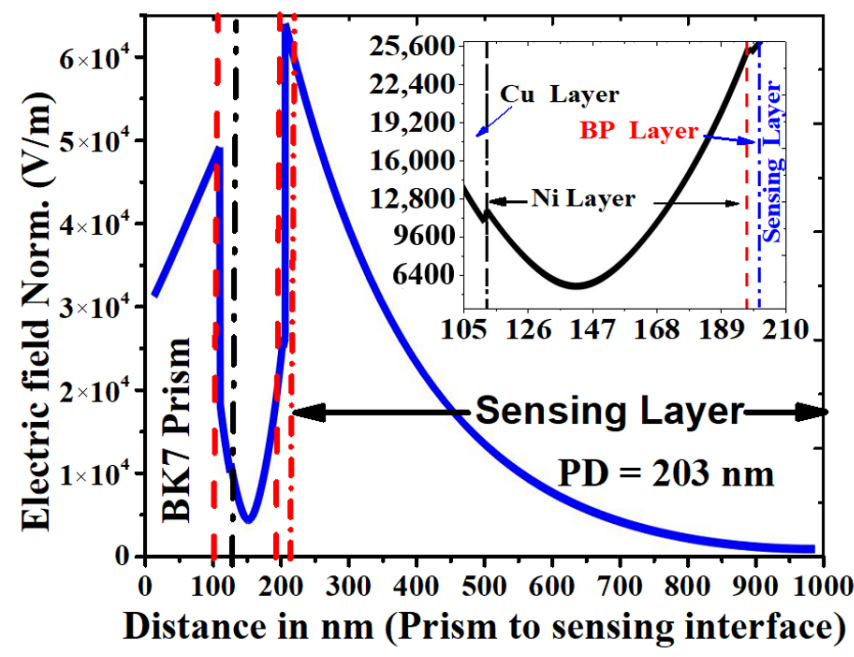

Figure 9. A normalized electric field $\left(E_{y}\right)$ is normal to the interface for the proposed sensor. The inset shows the enlarged view.

\section{Conclusions}

Relying on high adsorption energy, unique sensing ability, and the selectivity of phosphorene for cortisol concentration and benefitting from tunable performance arising from the anisotropy of phosphorene, we reported tunable and sensitive cortisol detection using a surface plasmon resonance sensor configuration. A bimetallic combination of $\mathrm{Cu}$ (35 nm) and Ni (20 nm) is used to obtain a strong electromagnetic field in the analyte medium. The sensor performance is evaluated in terms of sensitivity, the figure of merit 
(FOM), the limit of detection (LOD), and a new parameter called FOM* and penetration depth (PD). Our proposed sensor has a very high sensitivity of $343.78^{\circ} / \mathrm{RIU}, \mathrm{FOM}$ of 243.82 $\mathrm{RIU}^{-1}$, maximum FOM* of 1780.3 , sharper LOD of $0.026 \mathrm{ng} / \mathrm{mL}$, and a good PD of $203 \mathrm{~nm}$. All of these performance parameters significantly increase the efficiency of cortisol sensing. With developments in fabrication processes, we hope that the proposed research will pave the way for the creation of non-invasive, adjustable detection of cortisol concentrations in saliva in the near future.

Supplementary Materials: The following are available online at https:/ /www.mdpi.com/article/10 .3390 / magnetochemistry8030031/s1, SM1-Details about reflectance calculation for p-polarized light, SM2-Optimization of rotation angle $(\varphi)$ for different $\mathrm{Cu} / \mathrm{Ni}$ metal layer thickness combinations, Figures S1-S11.

Author Contributions: V.K.V.: simulation design, writing-original draft; S.P.: conceptualization, formal analysis, design, simulation, modeling, analysis; C.R.: supervision; Y.K.P.: conceptualization, supervision, design, analysis. All authors have read and agreed to the published version of the manuscript.

Funding: This research received no external funding.

Institutional Review Board Statement: Not applicable.

Informed Consent Statement: Not applicable.

Data Availability Statement: Correspondence and requests for any result of the manuscript should be addressed to yogendrapra@mnnit.ac.in.

Acknowledgments: Yogendra Kumar Prajapati gratefully acknowledges the DST-FIST, Government of India project (SR/FST/ETI-418/2016).

Conflicts of Interest: The authors declare no conflict of interest.

\section{References}

1. Stevens, R.C.; Soelberg, S.D.; Near, S.; Furlong, C.E. Detection of Cortisol in Saliva with a Flow-Filtered, Portable Surface Plasmon Resonance Biosensor System. Anal. Chem. 2008, 80, 6747-6751. [CrossRef]

2. Dhull, N.; Kaur, G.; Gupta, V.; Tomar, M. Highly sensitive and non-invasive electrochemical immunosensor for salivary cortisol detection. Sens. Actuators B Chem. 2019, 293, 281-288. [CrossRef]

3. Pandey, A.K.; Sharma, A.K.; Marques, C. On The Application of $\mathrm{SiO}_{2} / \mathrm{SiC}$ Grating on Ag for High-Performance Fiber Optic Plasmonic Sensing of Cortisol Concentration. Materials 2020, 13, 1623. [CrossRef] [PubMed]

4. Jung, H.; Jung, J.; Kim, Y.-H.; Kwon, D.; Kim, B.-G.; Bin Na, H.; Lee, H.H. Surface Plasmon Resonance Characteristics of au Nanoparticles Layered Sensor Chip for Direct Detection of Stress Hormone Conjugated by Nanoparticles. Biochip. J. 2018, 12, 249-256. [CrossRef]

5. Usha, S.P.; Shrivastav, A.M.; Gupta, B.D. A contemporary approach for design and characterization of fiber-optic-cortisol sensor tailoring LMR and ZnO/PPY molecularly imprinted film. Biosens. Bioelectron. 2017, 87, 178-186. [CrossRef] [PubMed]

6. Kämäräinen, S.; Mäki, M.; Tolonen, T.; Palleschi, G.; Virtanen, V.; Micheli, L.; Sesay, A.M. Disposable electrochemical immunosensor for cortisol determination in human saliva. Talanta 2018, 188, 50-57. [CrossRef]

7. Dalirirad, S.; Steckl, A.J. Aptamer-based lateral flow assay for point of care cortisol detection in sweat. Sens. Actuators B Chem. 2019, 283, 79-86. [CrossRef]

8. Chen, X.; Zhang, L.; Cui, D. Surface plasmon resonance immunoassay for cortisol determination with a self-assembling denaturalised bovine serum albumin layer on surface plasmon resonance chip. Micro Nano Lett. 2016, 11, 20-23. [CrossRef]

9. Frasconi, M.; Mazzarino, M.; Botrè, F.; Mazzei, F. Surface plasmon resonance immunosensor for cortisol and cortisone determination. Anal. Bioanal. Chem. 2009, 394, 2151-2159. [CrossRef] [PubMed]

10. Joa, S.; Lee, W.; Park, J.; Kim, W.; Kim, W.; Lee, G.; Lee, H.-J.; Hong, J.; Park, J. Localized surface plasmon resonance aptasensor for the highly sensitive direct detection of cortisol in human saliva. Sens. Actuators Chem. 2020, 304, 127424. [CrossRef]

11. Leitão, C.; Leal-Junior, A.; Almeida, A.R.; Pereira, S.O.; Costa, F.M.; Pinto, J.L.; Marques, C. Cortisol AuPd plasmonic unclad POF biosensor. Biotechnol. Rep. 2021, 29, e00587. [CrossRef]

12. Maurya, J.B.; Prajapati, Y.; Singh, V.; Saini, J.; Tripathi, R. Improved performance of the surface plasmon resonance biosensor based on graphene or $\mathrm{MoS}_{2}$ using silicon. Opt. Commun. 2016, 359, 426-434. [CrossRef]

13. Srivastava, T.; Jha, R. Black Phosphorus: A New Platform for Gaseous Sensing Based on Surface Plasmon Resonance. IEEE Photonics Technol. Lett. 2018, 30, 319-322. [CrossRef] 
14. Singh, M.K.; Pal, S.; Verma, A.; Prajapati, Y.K.; Saini, J.P. Highly sensitive antimonene-coated black phosphorous-based surface plasmon-resonance biosensor for DNA hybridization: Design and numerical analysis. J. Nanophoton. 2020, 14, 046015. [CrossRef]

15. Kumar, R.; Pal, S.; Verma, A.; Prajapati, Y.K.; Saini, J.P. Effect of silicon on sensitivity of SPR biosensor using hybrid nanostructure of black phosphorus and MXene. Superlattices Microstruct. 2020, 145, 106591. [CrossRef]

16. Pumera, M. Phosphorene and Black Phosphorus for Sensing and Biosensing. Trends Anal. Chem. 2017, 93, 1-6. [CrossRef]

17. Zhu, J.; Xiao, G.; Zuo, X. Two-Dimensional Black Phosphorus: An Emerging Anode Material for Lithium-Ion Batteries. Nano-Micro Lett. 2020, 12, 120. [CrossRef]

18. Cho, S.Y.; Prajapati, Y.; Singh, V.; Saini, J.; Tripathi, R. Superior Chemical Sensing Performance of Black Phosphorus: Comparison with $\mathrm{MoS}_{2}$ and Graphene. Adv. Mater. 2016, 28, 7020-7028. [CrossRef]

19. Pal, S.; Verma, A.; Prajapati, Y.K.; Saini, J.P. Influence of black phosphorous on performance of surface plasmon resonance biosensor. Opt. Quantum Electron. 2017, 49, 403. [CrossRef]

20. Khandelwal, A.; Mani, H.; Karigerasi, M.H.; Lahiri, I. Phosphorene-The two-dimensional black phosphorous: Properties, synthesis and applications. Mater. Sci. Eng. B 2017, 221, 17-34. [CrossRef]

21. Yasaei, P.; Behranginia, A.; Foroozan, T.; Asadi, M.; Kim, K.; Araghi, F.K.; Khojin, A.S. Stable and selective humidity sensing using stacked black phosphorus flakes. ACS Nano 2015, 9, 9898-9905. [CrossRef] [PubMed]

22. Singh, Y.; Raghuwanshi, S.K. Sensitivity Enhancement of the Surface Plasmon Resonance Gas Sensor with Black Phosphorus. IEEE Sens. Lett. 2019, 3, 1-4. [CrossRef]

23. Pal, S.; Verma, A.; Prajapati, Y.K.; Saini, J.P. Sensitive detection using heterostructure of black phosphorus, transition metal di-chalcogenides and MXene in SPR sensor. Appl. Phys. A 2020, 126, 809. [CrossRef]

24. Sharma, A.K. Plasmonic biosensor for detection of hemoglobin concentration in human blood: Design considerations. J. Appl. Phys. 2013, 114, 044701. [CrossRef]

25. Kumar, R.; Pal, S.; Pal, N.; Mishra, V.; Prajapati, Y.K. High-performance bimetallic surface plasmon resonance biochemical sensor using a black phosphorus-MXene hybrid structure. Appl. Phys. A 2021, 127, 259. [CrossRef]

26. Hossain, M.M.; Talukder, M.A. Gate-controlled graphene surface plasmon resonance sensor. Opt. Commun. 2021, $493,126994$. [CrossRef]

27. Singh, M.K.; Pal, S.; Verma, A.; Das, R.; Prajapati, Y.K. A nanolayered structure for sensitive detection of hemoglobin concentration using surface plasmon resonance. Appl. Phys. A 2021, 127, 832. [CrossRef] 